\title{
A Controllable Inflammatory Response and Temporary Abnormal Coagulation in Moderate Disease of COVID-19 in Wuhan, China
}

\author{
Yuchen Liu ${ }^{\mathrm{a}, \mathrm{c}}$, Xiaohong Zhang ${ }^{\mathrm{b}, \mathrm{c}}$, Jialu Qiao ${ }^{\mathrm{a}}$, Ruyu Gong ${ }^{\mathrm{b}}$, Qiang You ${ }^{\mathrm{a}}$, \\ Jian Sun ${ }^{\mathrm{a}}$, Wei Liu ${ }^{\mathrm{a}}$, Binlian Sun ${ }^{\mathrm{a}, \mathrm{d}}$
}

\begin{abstract}
Background: The coronavirus disease 2019 (COVID-19) is now a worldwide challenge for public health. Among 7 million patients, about $80 \%$ present mild to moderate disease, but studies dedicate to these patients are actually scarce. The aim of our study is to clarify the characteristics of laboratory test index of COVID-19 patient with moderate symptoms during the first wave of the pandemic in Wuhan, China.
\end{abstract}

Methods: In this retrospective cohort study, we included 107 adult inpatients with confirmed moderate disease of COVID-19 from the Affiliated Hospital of Jianghan University during February and early March 2020. All of these patients were recovered from COVID-19 and discharged from hospital. Demographic, clinical, and laboratory data of admission and discharge were extracted from electronic medical records and analyzed using SPSS, as well as among young, middle age and elderly people.

Results: The median age of this cohort of patients was 56.0 years. And the median hospitalization time was 16 days. Common clinical manifestations included fever, cough, asthenia and shortness of breath. On admission, laboratory results showed normal or increased neutrophil ratio, low lymphocyte count, decreased hemoglobin level, and increased inflammatory indicators (erythrocyte sedimentation rate (ESR) and C-reactive protein (CRP)); and some patients were complicated with coagulation disorder and myocardial damage. Furthermore, patients older than 60 years had statistically higher CRP, ESR and fibrinogen level. As the health condition was improved at discharge, the median level of most laboratory results were in the normal range except hemoglobin and related blood cell count, as well as

Manuscript submitted July 21, 2020, accepted July 31, 2020

Published online August 15, 2020

${ }^{a}$ Wuhan Institute of Biomedical Sciences, School of Medicine, Jianghan University, Wuhan, China

${ }^{\mathrm{b}}$ The Affiliated Hospital of Jianghan University, Wuhan, China

'The two authors contributed equally.

${ }^{\mathrm{d} C}$ Corresponding Author: Binlian Sun, Wuhan Institute of Biomedical Sciences, School of Medicine, Jianghan University, Wuhan 430056, China.

Email: binlian17@jhun.edu.cn

doi: https://doi.org/10.14740/jocmr4293 inflammatory indicator ESR. And patients older than 60 years showed slower recovery on coagulation parameters when compared to younger patients.

Conclusions: The severe acute respiratory syndrome coronavirus 2 (SARS-CoV-2) infection induces a controllable inflammatory response in moderate disease of COVID-19 in Wuhan, China. Since patients older than 60 years had higher inflammatory state and more dysregulated coagulation condition, it might be essential to closely assess their illness.

Keywords: COVID-19; Moderate disease; Clinical feature; Laboratory findings; Inflammatory

\section{Introduction}

The novel severe acute respiratory syndrome coronavirus 2 (SARS-CoV-2) was first identified in Wuhan, China in December 2019 [1,2], and now spread all over the world. Its infection in human mainly appears as acute respiratory syndrome, sometimes along with digestive and nervous disorders $[3,4]$. The outbreak of this coronavirus disease 2019 (COVID-19) was declared a pandemic by the World Health Organization (WHO) on March 11, 2020. Globally, as of June 28, 2020, there have been 9,843,073 confirmed cases including 495,760 deaths [5].

A large cohort study showed the spectrum of COVID-19 that among 44,415 patients, $81 \%$ had mild symptom, $14 \%$ had severe disease, and 5\% were in critical condition [6]. Naturally most studies about COVID-19 focus on the severe and critical cases, although more than $80 \%$ of COVID-19 patients show mild to moderate symptoms. It should be equally important to understand the average degree of SARS-CoV-2 infection, especially if this virus would become another endemic virus in communities like the influenza virus.

Our study describes 107 patients that were admitted in a designated hospital in Wuhan, the Affiliated Hospital of Jianghan University (the sixth hospital of Wuhan city) during February and early March 2020. These patients showed moderate symptom of COVID-19, and were confirmed both by 
SARS-CoV-2 RNA test and chest radiography. And they were all discharged from hospital after recovery. The aim of our study is to clarify the characteristics of laboratory test index of COVID-19 patient with moderate symptoms during the first wave of the pandemic in Wuhan. These findings may help us extend our understanding of the pathogenicity in SARS-CoV-2 infection.

\section{Materials and Methods}

\section{Patients}

This retrospective cohort study included adult patients $(\geq$ 18 years old) with confirmed COVID-19 admitted to the Affiliated Hospital of Jianghan University (the sixth hospital of Wuhan city) in Wuhan from February 1 to March 5, 2020. According to WHO interim guidance [7], 107 patients with moderate disease of COVID-19 on admission were enrolled in this study; they showed clinical signs of pneumonia (fever, cough, dyspnea, fast breathing) but no signs of severe pneumonia, including oxygen saturation $\left(\mathrm{SpO}_{2}\right) \geq 90 \%$ on room air. All the patients were confirmed by SARS-CoV-2 RNA test in respiratory secretions for twice, as well as by ground-glass opacities or bilateral pulmonary infiltration showed in chest computed tomography (CT) scan. During hospitalization, patients were kept in regular wards without intensive cares or invasive mechanical ventilation. They received supportive therapy, effective oxygen therapy, antiviral agent and, if necessary, antibiotics. Patients were discharged from hospital when the following criteria were met: body temperature normal for more than 3 days, respiratory symptoms significantly improved, pulmonary imaging significantly improved on CT scan, and SARS-CoV-2 RNA tests showed negative for twice. This study was approved by the Ethics Committee of School of Medicine of Jianghan University (Wuhan, China). This study was conducted in compliance with the ethical standards of the responsible institution on human subjects as well as with the Helsinki Declaration.

\section{Data collections}

The laboratory tests including blood routine, biochemistry, coagulation parameters, and cardiac injury biomarkers were performed in patients on admission and during the hospitalization. The demographic and clinical information, laboratory results, and outcome data were finally collected from electronic medical records.

\section{Statistical analysis}

Continuous variables were expressed as median (interquartile range (IQR)) and compared with the one-way analysis of variance (ANOVA) test between different age groups. A two-sided $\alpha$ of less than 0.05 was considered statistically significant. Statistical analyses were done using SPSS (ver-
Table 1. Demographics and Clinical Characteristics of Patients on Admission ( $\mathrm{N}=107$ )

\begin{tabular}{|c|c|}
\hline Age (median (IQR), year) & $56(43.5-65.0)$ \\
\hline \multicolumn{2}{|l|}{ Sex } \\
\hline Female & $62(58 \%)$ \\
\hline Male & $45(42 \%)$ \\
\hline Hospitalization days (median (IQR), day) & $16(13-20.5)$ \\
\hline \multicolumn{2}{|l|}{ Medical history } \\
\hline Hypertension & $31(29 \%)$ \\
\hline Diabetes & $6(5.6 \%)$ \\
\hline Heart disease & $10(9.3 \%)$ \\
\hline Cancer & $3(2.8 \%)$ \\
\hline Other respiratory disease & $6(5.6 \%)$ \\
\hline Kidney disease & $6(5.6 \%)$ \\
\hline Liver disease & $6(5.6 \%)$ \\
\hline Neurological disease & $1(0.9 \%)$ \\
\hline Thyroid disease & $2(1.9 \%)$ \\
\hline Others & $8(7.5 \%)$ \\
\hline \multicolumn{2}{|l|}{ Symptoms } \\
\hline Fever & $84(78.5 \%)$ \\
\hline Cough & $63(58.9 \%)$ \\
\hline Sputum production & $18(16.8 \%)$ \\
\hline Nose obstruction, rhinorrhea & $2(1.9 \%)$ \\
\hline Shortness of breath & $32(29.9 \%)$ \\
\hline Headache & $5(4.7 \%)$ \\
\hline Chest pain & $2(1.9 \%)$ \\
\hline Myalgia & $10(9.3 \%)$ \\
\hline Asthenia & $35(32.7 \%)$ \\
\hline Vomiting & $3(2.8 \%)$ \\
\hline Diarrhea & $12(11.2 \%)$ \\
\hline \multicolumn{2}{|l|}{ Signs } \\
\hline Respiratory rate (median (IQR), bpm) & $23(21-30)$ \\
\hline Heart rate (median (IQR), bpm) & $88(82.5-98)$ \\
\hline Systolic pressure $>140 \mathrm{~mm} \mathrm{Hg}$ & $18(17 \%)$ \\
\hline $\mathrm{SpO}_{2} \leq 93 \%$ & $27(25 \%)$ \\
\hline
\end{tabular}

Data are median (IQR), $\mathrm{n}(\%)$, or $\mathrm{n} / \mathrm{N}(\%)$. IQR: interquartile range; $\mathrm{SpO}_{2}$ : oxygen saturation.

sion 19.0, IBM).

\section{Results}

\section{Patient demographics and characteristics}

A total of 107 patients with moderate disease of COVID-19 were recruited. The demographic and clinical characteristics 
Table 2. Summary of Laboratory Findings on Admission and at Discharge

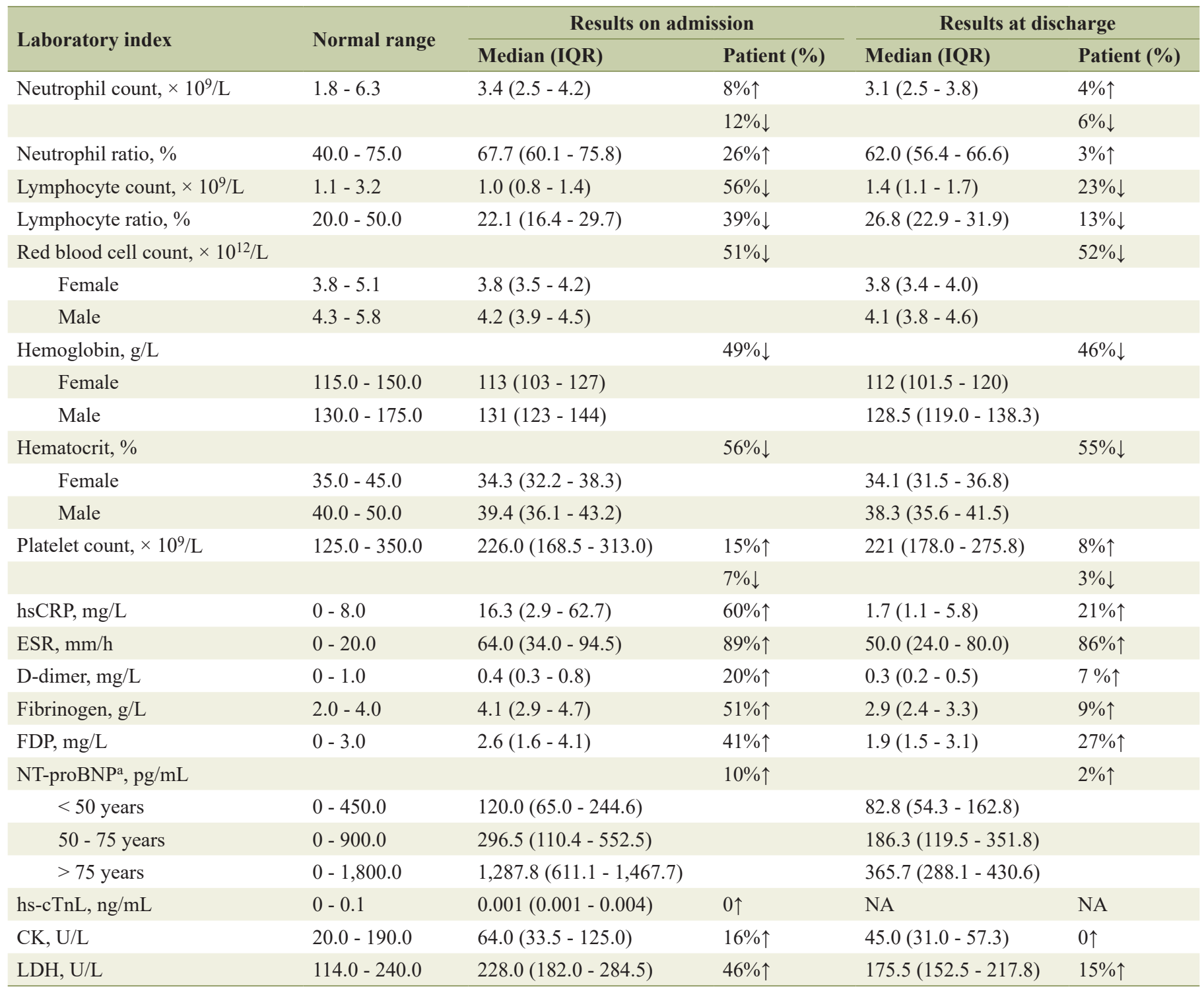

Data are median (IQR) or n/N (\%). aThe normal level of NT-proBNP is increased with age. Three normal ranges for different age groups are listed, and results are presented accordingly. $\uparrow$ For increased blood level. $\downarrow$ For decreased blood level. hsCRP: high sensitive C-reactive protein; ESR: erythrocyte sedimentation rate; FDP: fibrinogen degradation product; NT-proBNP: N-terminal prohormone brain natriuretic peptide; hs-cTnL: high sensitive cardiac troponin L; CK: creatine kinase; LDH: lactose dehydrogenase; IQR: interquartile range; NA: not applicable.

of these patients are shown in Table 1. Of these patients (62 females and 45 males), the median age at disease onset was 56 years (range: 20 - 92 years), with 40 patients (37\%) older than 60 years. Most patients had fever (78.5\%) and cough $(58.9 \%)$ as their first symptoms; some also had asthenia $(32.7 \%)$, shortness of breath $(29.9 \%)$, sputum production $(16.8 \%)$ and diarrhea $(11.2 \%)$. As for the underlying diseases, hypertension $(29 \%)$, heart disease $(9.3 \%)$ and diabetes $(5.6 \%)$ were the most common in medical histories of these patients. On admission, the vital signs of patients were also recorded. Notably, although the $\mathrm{SpO}_{2}$ of all the patients were $\geq 90 \%$ at room air, $25 \%$ of patients were $\leq 93 \%$. Patients received symptomatic and pneumonia treatments in hospital; and the median of their hospitalization were 16 days (IQR: $13-20)$.

\section{Whole blood cell counting findings}

The whole blood cell counting was monitored for all the patients on admission and during their hospitalization (median values showed in Table 2). The white blood cell counts were generally in the normal range, whereas $56 \%$ of patients (60 of 107 patients) showed lymphopenia (lymphocyte count $<1.1$ $\times 10^{9} / \mathrm{L}$, Table 2 ). Among patients showed lymphopenia, $57 \%$ (34 of 60) had a decrease by less than 30\% (lymphocyte count 
a

Decreased Lymphocyte count

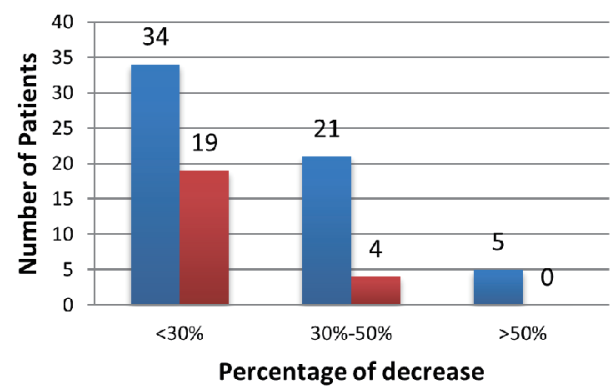

C

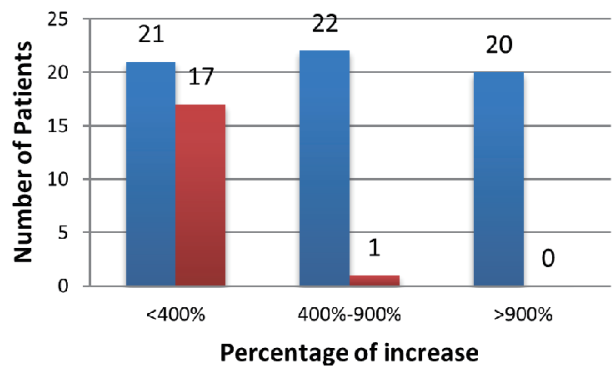

e

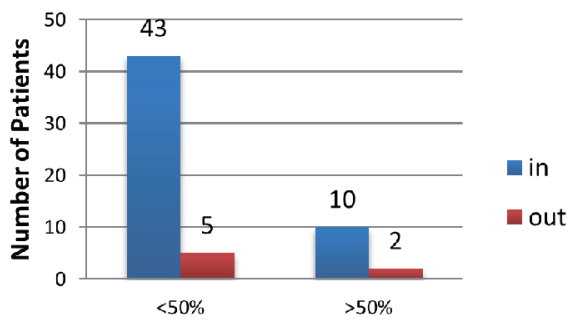

Percentage of increase

g

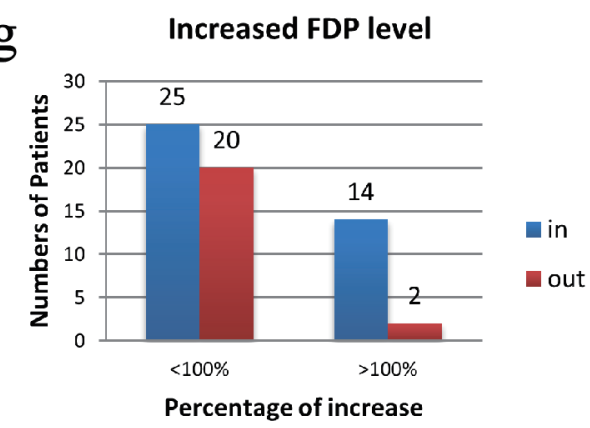

\section{b Decreased Hemoglobin level}
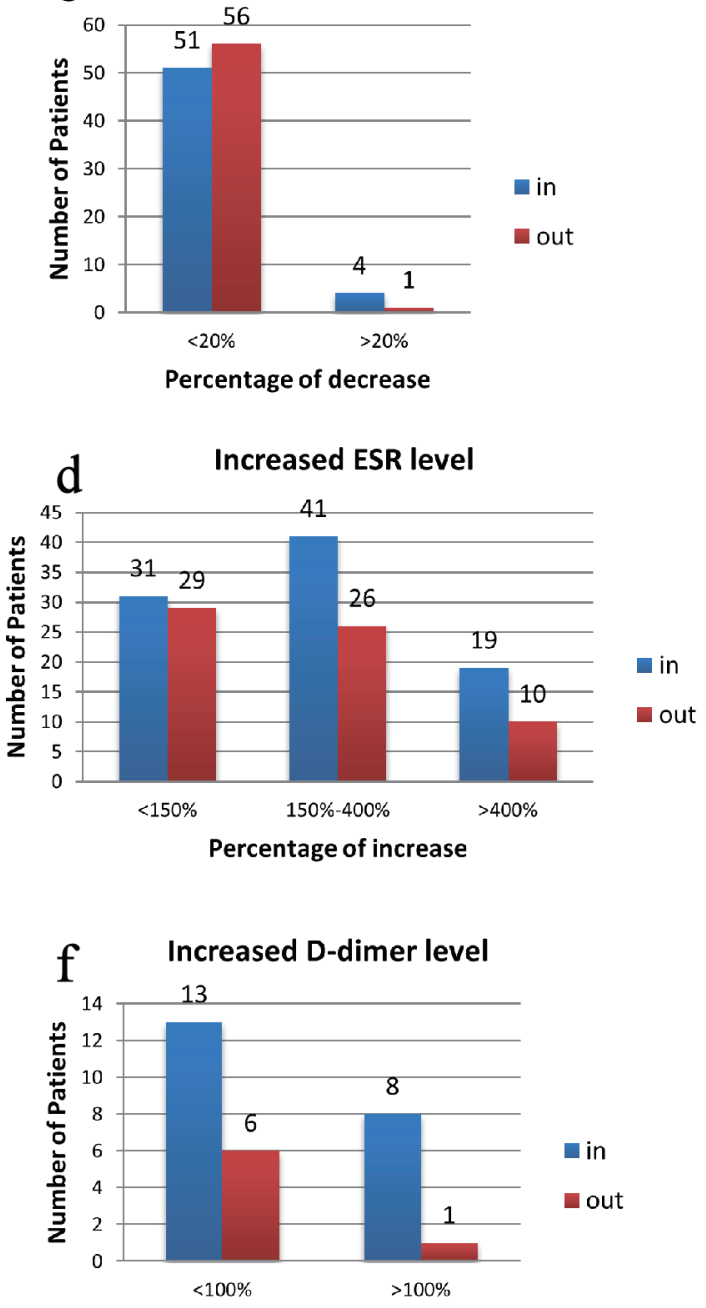

Percentage of increase

Figure 1. Illustration of percentage changes of laboratory index according to number of patients. Data are presented as number of patients that had changed level in laboratory assessments. (a) Decreased lymphocyte count on admission and at discharge. (b) Decreased hemoglobin level on admission and at discharge. (c) Increased CRP level on admission and at discharge. (d) Increased ESR level on admission and at discharge. (e) Increased fibrinogen level on admission and at discharge. (f) Increased D-dimer level on admission and at discharge. (g) Increased FDP level on admission and at discharge. In for the data collected on admission, out for data collected at discharge. CRP: C-reactive protein; ESR: erythrocyte sedimentation rate; FDP: fibrinogen degradation product. 
Table 3. Statistically Significant Biomarkers on Admission and at Discharge With Different Ages

\begin{tabular}{llll} 
& $<\mathbf{4 5}(\mathbf{n}=\mathbf{2 9})$ & $\mathbf{4 5}-\mathbf{6 0}(\mathbf{n}=\mathbf{3 8})$ & $>\mathbf{6 0}(\mathbf{n}=\mathbf{4 0})$ \\
\hline CRP (in) & $7.2(0.7-53.2)$ & $15.1(2.5-44.3)$ & $51.9(6.5-94.4)^{\mathrm{a}}$ \\
ESR (in) & $35.0(23.0-65.0)$ & $53.0(32.5-90.5)$ & $87.5(56.0-107.3)^{\mathrm{a}}$ \\
ESR (out) & $23.0(21.0-46.0)$ & $42.0(25.0-63.0)$ & $71.0(34.5-98.3)^{\mathrm{a}}$ \\
Fibrinogen (in) & $3.6(2.2-4.7)$ & $4.1(2.8-4.6)$ & $4.4(3.5-5.3)^{\mathrm{b}}$ \\
FDP (out) & $1.7(1.4-2.3)$ & $1.8(1.4-2.2)$ & $2.9(1.9-4.1)^{\mathrm{a}}$ \\
D - dimer (out) & $0.3(0.2-0.4)$ & $0.3(0.2-0.4)$ & $0.49(0.32-0.82)^{\mathrm{a}}$ \\
CK & $79.0(50.0-161.0)$ & $78.5(33.3-135.3)$ & $49.0(30.8-79.8)^{\mathrm{b}}$ \\
\hline
\end{tabular}

Data are expressed with the median (IQR). P values comparing different age groups are from one-way ANOVA. aP $<0.05$ for $45-60$ group and $<45$ group vs. $>60$ group has statistical difference. ${ }^{\text {bP }}<0.05$ for $<45$ group vs. $>60$ group has statistical difference. In for the data collected on admission, out for data collected at discharge. CRP: C-reactive protein; ESR: erythrocyte sedimentation rate; FDP: fibrinogen degradation product; CK: creatine kinase; IQR: interquartile range.

$>0.8 \times 10^{9} / \mathrm{L}$; Fig. 1a) while $8 \%$ (five of 60 ) of them showed a decrease by more than $50 \%$ (lymphocyte count $<0.5 \times 10^{9} / \mathrm{L}$; Fig. 1a). Twenty-three percent (23 of 98) of patients (Table 2) still had lymphopenia when they were discharged from hospital, and the decrease was limited for most (Fig. 1a). The change of neutrophil count affects fewer patients on admission, $8 \%$ of (nine of 107) patients showed neutrophilia (neutrophil count $>$ $6.3 \times 10^{9} / \mathrm{L}$, Table 2 ) and $12 \%$ (13 of 107) patients had neutropenia (neutrophil count $<3.5 \times 10^{9} / \mathrm{L}$, Table 2). However, neutrophil ratio was augmented in $26 \%$ (28 of 107) of patients (neutrophil ratio $>75 \%$, Table 2 ), and the maximum increase was about $20 \%$. This ratio appeared to be in the normal range for most patients at discharge. Therefore, the main change in white blood cell counts for patients with moderate COVID-19 was the decrease of lymphocyte. And as the health condition improved at discharge, lymphocyte count also gradually increased to normal level.

The data showed that more than $50 \%$ of all patients had declined level of red blood cell count (55 of 107), hemoglobin (52 of 107) and hematocrit (60 of 107) (Table 2); and four female patients were in severe condition (hemoglobin $<90 \mathrm{~g} / \mathrm{L}$, Fig. 1b). When discharged from hospital, 50\% (45 of 98) of patients still had these decreases (Table 2). The four female patients mentioned above had their hemoglobin increased to over $90 \mathrm{~g} / \mathrm{L}$. However, one female of 70 years had less than $90 \mathrm{~g} / \mathrm{L}$ hemoglobin and that was less than earlier result. Furthermore, there were five more male patients had hemoglobin declined at discharge (Fig. 1b). These results suggested that SARS-CoV-2 infection may be accompanied by oxygen transport defect in red blood cells.

As for the platelet counting on admission, $7 \%$ of patients (seven of 107) had decreased platelet level (platelet count $<$ $125 \times 10^{9} / \mathrm{L}$, Table 2), and $15 \%$ (16 of 107) had increased level (platelet count $>350 \times 10^{9} / \mathrm{L}$, Table 2 ). At discharge, most patients showed platelet level in the normal range (Table 2).

\section{Inflammatory biomarkers}

Inflammatory biomarkers were also examined on admission and during hospitalization, such as erythrocytes sedimentation rate (ESR), C-reactive protein (CRP) and procalcitonin (PCT). For most patients, PCT levels were in the normal range (data not shown). For CRP, the median value was $16.3 \mathrm{mg} / \mathrm{L}$ (IQR: 2.9 - 62.7) among 105 patients on admission (Table 2), and patients older than 60 years had significantly higher level (Table 3 ). To be specific, CRP levels in serum were increased (CRP $>8 \mathrm{mg} / \mathrm{L}$, Table 2 ) in $60 \%$ of patients (63 of 105 ), and $40 \%$ (42 of 105) showed an increase by more than 400\% (CRP $>40$ $\mathrm{mg} / \mathrm{L}$, Fig. 1c). At discharge, only $21 \%$ (18 of 87 , Table 2 ) had elevated level of CRP, and the increased level did not exceed $40 \mathrm{mg} / \mathrm{L}$ except for one patient (Fig. 1c).

Levels of ESR were increased in $89 \%$ of patients (91 of 102, ESR $>20 \mathrm{~mm} / \mathrm{h}$, Table 2), and 59\% (60 of 102, Fig. 1d) had an increase exceeding $50 \mathrm{~mm} / \mathrm{h}$. Similar to CRP, patients older than 60 years had statistically higher ESR level (Table 3 ). The median value of ESR on admission was $64 \mathrm{~mm} / \mathrm{h}$ (IQR: 34.0 - 94.5), while the value improved to $50 \mathrm{~mm} / \mathrm{h}$ (IQR: 24.0 - 80.0) at discharge (Table 2). There were still 36 patients had ESR level more than $50 \mathrm{~mm} / \mathrm{h}$ in serum (Fig. 1d).

\section{Coagulation parameters}

The coagulation parameters were examined on admission for 103 patients. Eighty percent of patients (82 of 103, Table 2) had normal serum levels of D-dimer on admission. Among the $20 \%$ of patients (21 of 103) who had increased D-dimer level (D-dimer $>1 \mathrm{mg} / \mathrm{L}$, Table 2), eight showed an increase by more than 100\% (D-dimer $>2 \mathrm{mg} / \mathrm{L}$, Fig. 1f). At discharge, 7\% of patients (seven of 94) still had abnormal level of D-dimer, though the results were very close to the normal range. The fibrinogen level, on the other hand, had increased in $51 \%$ of patients ( 53 of 103, fibrinogen $>4 \mathrm{mg} / \mathrm{L}$, Table 2$)$ and decreased in $14 \%$ (14 of 103 , fibrinogen $<2 \mathrm{mg} / \mathrm{L})$. Specifically, $10 \%$ patients (10 of 103) had an increase of fibrinogen level by more than $50 \%$ (fibrinogen $>6 \mathrm{mg} / \mathrm{L}$, Fig. 1e), while most decreases were slight. Furthermore, when compared to patients younger than 45 years, fibrinogen level on admission was significantly higher in the patients older than 60 years (Table 3 ). At discharge, only seven patients still showed high level of fibrinogen (Fig. 1e). Fibrinogen degradation product (FDP) was also 
examined, and $41 \%$ of patients had elevated level (39 of 94, FDP $>3 \mathrm{mg} / \mathrm{L}$, Table 2, Fig. 1g) on admission. Twenty-seven percent of patients (22 of 83) still showed increased amount of FDP at discharge (Table 2). Therefore, fibrinogen and FDP were the most altered parameters in coagulation function from our analysis.

\section{Cardiac injury biomarkers}

Cardiac injury biomarkers were monitored for patients on admission. Among the 106 patients assessed, all had normal serum levels of high sensitive cardiac troponin L (hs-cTnL, $<0.1 \mathrm{ng} / \mathrm{mL}$ ). The other cardiac biomarker examined was Nterminal prohormone brain natriuretic peptide (NT-proBNP), and $10 \%$ of patients (10 of 103) had elevated level (NT-proB$\mathrm{NP}>1,800,900$, and $450 \mathrm{pg} / \mathrm{mL}$ according to age, Table 2) on admission, and 5\% (five of 103) had an increase by more than $200 \%$. At discharge, the NT-proBNP level had decreased into the normal range except two male patients. These two patients still had high level of NT-proBNP (more than 1,000 pg/ $\mathrm{mL}$ ), however, other cardiac biomarkers such as hs-cTnL and creatine kinase-MB (CK-MB) were both in the normal range. Considering their history of hypertension, they were asked to reevaluate their heart function after the COVID-19.

\section{Discussion}

As an emerging infectious disease, COVID-19 is now a worldwide challenge for public health. Among the 7 million patients, about $80 \%$ present mild to moderate disease, but studies dedicate to these patients are actually scarce. Therefore, we enrolled 107 adult patients with moderate disease of COVID-19 to elucidate the clinical manifestation and laboratory findings.

One of the risk factors of critical and mortal COVID-19 is male with older age [8]. Considering the not severe condition of disease, it is rational that female patients (58\%) present more than male in this cohort. Previous studies have found comorbidity such as diabetes and hypertension, heart disease as other risk factors [9]. Similar to other study, 5.6\% of patients had diabetes and 29\% had hypertension. Similar to other studies in Asia $[10,11]$, patients mainly present fever $(78.5 \%)$, cough $(58.9 \%)$, asthenia $(32.7 \%)$ and shortness of breath $(29.9 \%)$. In Europe, however, a recent study showed that patients with mild to moderate disease mainly had headache, loss of smell, nasal obstruction and asthenia [12]. Considering the big differences, it is possible that olfactory dysfunction might be neglected during the first wave of SARS-CoV-2 infection in Wuhan. Also, the virus is phylogenetically distinct from Asia (type B) to Europe (type C) [13], which could contribute to different clinical outcomes.

Through analysis of laboratory findings, we found that patients with moderate disease of COVID-19 had common characteristics on admission: normal or increased neutrophil ratio, low lymphocyte count, decreased hemoglobin level, increased inflammatory indicators (ESR and CRP); and some patients were complicated with coagulation disorder and myo- cardial damage. Since the outcome of this cohort is known as recovery and discharge from hospital, we also collected the last available laboratory results. Although all the patients met the discharge criteria for COVID-19, some still had abnormal laboratory findings. Especially, we found the levels of the inflammatory biomarker ESR were still elevated for half of the patients tested. Hence, patients were asked to go back to the hospital for follow-up examination including the virus RNA test, chest CT scan, blood routine, coagulation function and other biochemical indicators on both the 14th and 28th day after discharge.

Previous studies have found that white blood cell (WBC), procalcitonin (PCT), aspartate aminotransferase (AST), lactose dehydrogenase (LDH), Cr, hs-cTnL, and D-dimer could indicate the progression of COVID-19 on admission, especially for the severe and mortal cases $[3,14,15]$. And in the moderate condition we analyzed, most patients had these indicators in the normal range. Therefore, our findings are consistent with other studies.

However, these biomarkers were not all specific to COVID-19. Similar to other virus infections including SARS [16] and H1N1 influenza [17], the neutrophil count was mostly normal in mild to moderate patients, while lymphocyte count was significantly decreased. As the major antiviral cells, lymphocyte count declined down to $0.27 \times 10^{9} / \mathrm{L}$ in this cohort on admission. And after receiving antiviral and supportive treatment, the lymphocyte counts increased among all the patients at discharge. Previous study found that lymphocyte counts would continually decrease in severe and critical COVID-19 patients [4]. These results suggested that SARS-CoV-2 infection can suppress the level of lymphocyte. Therefore, closely monitoring lymphocyte counts could be one of the best methods when we evaluate the outcome of moderate patients.

The blood routine results also showed that about $50 \%$ of patients had low level of hemoglobin, red blood cell, and hematocrit, and the condition was not improved at discharge. Previous studies also reported that $51 \%$ of 99 patients had hemoglobin below the normal range [18], and the hemoglobin level of severe patients was lower, although the difference was not statistically significant [3]. There might be two explanations. First, the inflammatory state caused by infection may interfere with erythrocyte/bone marrow metabolism and iron regulation [19], and eventually result in a decline of hemoglobin and red blood cell. Second, patients could suffer from anemia for some time, and appear more vulnerable to SARS-CoV-2 infection. Once infected, however, correction of anemia could not benefit from all the symptoms of COVID-19 and psychological stresses. The decrease of functioning hemoglobin may contribute to hypoxia, and further aggravate the disease in severe cases.

Examination of inflammatory biomarkers found that PCT was in the normal range for moderate patients, while CRP and ESR were increased in $60 \%$ and $90 \%$ of patients on admission, respectively. Other studies including mild to moderate disease showed heterogeneous results [20], and found 33\% (three of 10 ) or $100 \%$ (30 of 30) of patients had increased level of CRP $[21,22]$. Thus, SARS-CoV-2 infection is involved with the disorder of inflammatory response. Furthermore, we find that the increase of both CRP and ESR were correlated with patient's age, which may indicate that high inflammatory state 
and more severe pneumonia present more often in elderly people. In addition, the increase of CRP level was more transient, as it returned to normal range in most people when they were discharged from hospital. Nevertheless, it took more time for ESR to return to normal level, especially for patients older than 60 years. Certainly, elder people would need more time to recover and to metabolize the extra inflammatory biomarkers.

Abnormal coagulation condition is known as associated with poor prognosis of COVID-19. In particular, serum level of D-dimer and FDP were significantly higher in lethal cases [23]. Other coronavirus infection such as SARS and Middle East respiratory syndrome (MERS) showed similar increased coagulation activities in severe cases $[24,25]$. The possible mechanism is that local inflammatory response induced by cytokine responses stimulates coagulation cascade and hemodynamic changes $[26,27]$. In patients with moderate COVID-19, we found about $50 \%, 30 \%$ and $20 \%$ presented increased level in fibrinogen, FDP and D-dimer on admission, respectively. And when compared to young people, patients older than 60 years tend to have higher levels of these coagulation parameters. Certainly, the hemodynamics and endothelial conditions are more complicate in elder people. Furthermore, SARS-CoV-2 infection as well as the inflammatory response it induced could deteriorate the existed dysregulation. As the health condition improved at discharge, these parameters decreased to normal ranges for most patients. Yet again, patients over 60 years had significantly higher level of fibrin degradation products. In the meantime, we found that the platelet level had no obvious decrease in most patients with moderate disease. Therefore, as reported previously, thrombocytopenia happens more often in severe and critical patients [28], while abnormal coagulation condition is slight and temporary in moderate patients.

The receptor of SARS-CoV-2, angiotensin-converting enzyme 2 (ACE2) is expressed in myocardial cells and vascular endothelial cells [29]. It is possible that heart dysfunction is directly targeted by virus infection, hence early evaluation and continued monitoring of cardiac damage are important [30]. We systematically examined the cardiac function biomarkers on admission. CK-MB and cTnL had no obvious change in these moderate patients, while about $10 \%$ of patients showed increased NT-proBNP. This percentage is much lower than previous reported $27.5 \%$ of the severe cases that had elevated NT-proBNP. Furthermore, we only found two patients still had NT-proBNP higher than normal range at discharge, indicating that moderate patients had little cardiac complication.

This study provides us more information about moderate COVID-19, but still has some limitations. First, this is a relatively small, single-center study. Second, due to the retrospective study design, a few laboratory tests were not done in all patients. In addition, several patients were hospitalized during a short period that some tests were not re-examined before they were discharged. Third, we do not possess any viral kinetic data in these patients, further studies are necessary to elucidate the correlation between viral load and laboratory changes.

In this ongoing pandemic, $40 \%$ of infected people showed moderate disease [7], it is important to understand more about this moderate spectrum of disease to settle into a long-term problem. We conducted a cohort study of 107 adult patients with moderate disease of COVID-19 in Wuhan, China. Based on the clinical characteristics, we conclude that SARS-CoV-2 infection inhibits the immune system of patients, and induces a controllable inflammatory response in moderate COVID-19. Elderly patients have higher inflammatory state and more dysregulated coagulation condition. It is essential to closely assess their condition for a better clinical management.

\section{Acknowledgments}

This study was supported by the Natural Science Foundation of China (31670167), Hubei Province Department of Education Science and Technology Project (B2019232) and the Wuhan Municipal Education Bureau Project (2019003).

\section{Financial Disclosure}

There are no financial conflicts of interest to disclose.

\section{Conflict of Interest}

All authors declare no competing interests.

\section{Informed Consent}

All subjects provided written informed consent.

\section{Author Contributions}

YL, XZ and BS designed and performed the study. YL and BS drafted the manuscript and did critical editing. XZ and RG were involved in data collection; YL, JQ, QY, JS and WL analyzed the data. BS carefully supervised this manuscript preparation and writing.

\section{Data Availability}

All data used in the study are available from the corresponding author by request. Although some data confidential in nature may only be provided with restrictions

\section{References}

1. Zhu N, Zhang D, Wang W, Li X, Yang B, Song J, Zhao X, et al. A novel coronavirus from patients with pneumonia in China, 2019. N Engl J Med. 2020;382(8):727-733.

2. Coronaviridae Study Group of the International Committee on Taxonomy of Viruses. The species Severe acute respiratory syndrome-related coronavirus: classifying 2019-nCoV and naming it SARS-CoV-2. Nat Microbiol. 2020;5(4):536-544.

3. Huang C, Wang Y, Li X, Ren L, Zhao J, Hu Y, Zhang 
L, et al. Clinical features of patients infected with 2019 novel coronavirus in Wuhan, China. Lancet. 2020;395(10223):497-506.

4. Wang D, Hu B, Hu C, Zhu F, Liu X, Zhang J, Wang B, et al. Clinical characteristics of 138 hospitalized patients with 2019 novel coronavirus-infected pneumonia in Wuhan, China. JAMA. 2020;323(11):1061-1069.

5. WHO. Coronavirus disease (COVID-19) Situation Report - 160. Available from: https://www.who.int/docs/ default-source/coronaviruse/situation-reports/20200628covid-19-sitrep-160.pdf?sfvrsn=2fe1c658 2.

6. Wu Z, McGoogan JM. Characteristics of and important lessons from the coronavirus disease 2019 (COVID-19) outbreak in China: summary of a report of 72314 cases from the Chinese Center for Disease Control and Prevention. JAMA. 2020;323(13):1239-1242.

7. WHO. Clinical management of COVID19. Available from: https://www.who.int/publications-detail/clinicalmanagement-of-covid-19.

8. Liu K, Chen Y, Lin R, Han K. Clinical features of COVID-19 in elderly patients: A comparison with young and middle-aged patients. J Infect. 2020;80(6):e14-e18.

9. Zheng Z, Peng F, Xu B, Zhao J, Liu H, Peng J, Li Q, et al. Risk factors of critical \& mortal COVID-19 cases: A systematic literature review and meta-analysis. J Infect. 2020;81(2):e16-e25.

10. Li LQ, Huang T, Wang YQ, Wang ZP, Liang Y, Huang TB, Zhang HY, et al. COVID-19 patients' clinical characteristics, discharge rate, and fatality rate of meta-analysis. J Med Virol. 2020;92(6):577-583.

11. Cao Y, Liu X, Xiong L, Cai K. Imaging and clinical features of patients with 2019 novel coronavirus SARSCoV-2: A systematic review and meta-analysis. J Med Virol. 2020

12. Lechien JR, Chiesa-Estomba CM, Place S, Van Laethem Y, Cabaraux P, Mat Q, Huet K, et al. Clinical and epidemiological characteristics of 1420 European patients with mild-to-moderate coronavirus disease 2019. J Intern Med. 2020.

13. Forster P, Forster L, Renfrew C, Forster M. Phylogenetic network analysis of SARS-CoV-2 genomes. Proc Natl Acad Sci U S A. 2020;117(17):9241-9243.

14. Zhou F, Yu T, Du R, Fan G, Liu Y, Liu Z, Xiang J, et al. Clinical course and risk factors for mortality of adult inpatients with COVID-19 in Wuhan, China: a retrospective cohort study. Lancet. 2020;395(10229):1054-1062.

15. Velavan TP, Meyer CG. Mild versus severe COVID-19: Laboratory markers. Int J Infect Dis. 2020;95:304-307.

16. Lee N, Hui D, Wu A, Chan P, Cameron P, Joynt GM, Ahuja A, et al. A major outbreak of severe acute respiratory syndrome in Hong Kong. N Engl J Med. 2003;348(20):19861994.

17. Cheng Y, Zhao H, Song P, Zhang Z, Chen J, Zhou YH. Dynamic changes of lymphocyte counts in adult patients with severe pandemic H1N1 influenza A. J Infect Public Health. 2019;12(6):878-883.

18. Chen N, Zhou M, Dong X, Qu J, Gong F, Han Y, Qiu Y, et al. Epidemiological and clinical characteristics of 99 cases of 2019 novel coronavirus pneumonia in Wuhan, China: a descriptive study. Lancet. 2020;395(10223):507513.

19. Cavezzi A, Troiani E, Corrao S. COVID-19: hemoglobin, iron, and hypoxia beyond inflammation. A narrative review. Clin Pract. 2020;10(2):1271.

20. Zeng F, Huang Y, Guo Y, Yin M, Chen X, Xiao L, Deng G. Association of inflammatory markers with the severity of COVID-19: A meta-analysis. Int J Infect Dis. 2020;96:467-474

21. Chen G, Wu D, Guo W, Cao Y, Huang D, Wang H, Wang $\mathrm{T}$, et al. Clinical and immunological features of severe and moderate coronavirus disease 2019. J Clin Invest. 2020;130(5):2620-2629.

22. Wang F, Hou H, Luo Y, Tang G, Wu S, Huang M, Liu W, et al. The laboratory tests and host immunity of COVID-19 patients with different severity of illness. JCI Insight. 2020;5(10).

23. Tang N, Li D, Wang X, Sun Z. Abnormal coagulation parameters are associated with poor prognosis in patients with novel coronavirus pneumonia. J Thromb Haemost. 2020;18(4):844-847.

24. Wong RS, Wu A, To KF, Lee N, Lam CW, Wong CK, Chan PK, et al. Haematological manifestations in patients with severe acute respiratory syndrome: retrospective analysis. BMJ. 2003;326(7403):1358-1362.

25. Al-Abdallat MM, Payne DC, Alqasrawi S, Rha B, Tohme RA, Abedi GR, Al Nsour M, et al. Hospital-associated outbreak of Middle East respiratory syndrome coronavirus: a serologic, epidemiologic, and clinical description. Clin Infect Dis. 2014;59(9):1225-1233.

26. Moore JB, June $\mathrm{CH}$. Cytokine release syndrome in severe COVID-19. Science. 2020;368(6490):473-474.

27. Giannis D, Ziogas IA, Gianni P. Coagulation disorders in coronavirus infected patients: COVID-19, SARS-CoV-1, MERS-CoV and lessons from the past. J Clin Virol. 2020;127:104362.

28. Guan WJ, Ni ZY, Hu Y, Liang WH, Ou CQ, He JX, Liu $\mathrm{L}$, et al. Clinical Characteristics of Coronavirus Disease 2019 in China. N Engl J Med. 2020;382(18):1708-1720.

29. Mendoza-Torres E, Oyarzun A, Mondaca-Ruff D, Azocar A, Castro PF, Jalil JE, Chiong M, et al. ACE2 and vasoactive peptides: novel players in cardiovascular/renal remodeling and hypertension. Ther Adv Cardiovasc Dis. 2015;9(4):217-237.

30. Guzik TJ, Mohiddin SA, Dimarco A, Patel V, Savvatis K, Marelli-Berg FM, Madhur MS, et al. COVID-19 and the cardiovascular system: implications for risk assessment, diagnosis, and treatment options. Cardiovasc Res. 2020;116(10):1666-1687. 